\section{Cup-to-disc and arteriole-to-venule ratios in preterm birth}

\author{
J Kim ${ }^{1,2}$, DY Choi ${ }^{2}$, K-A Park ${ }^{2}$ and SY Oh
}

Abstract

Aims To investigate the influence of preterm birth on the optic disc and retinal vessels by measurements of cup-to-disc (C/D) ratio and arteriole-to-venule $(\mathrm{A} / \mathrm{V})$ ratio.

Methods Eighty-three eyes of 42 preterm births were included. In the age- and sexmatched control group, 83 eyes of 42 full-term births were used. Fundus color photographs were taken. ImageJ software was used to calculate $C / D$ and $A / V$ ratios from the fundus images.

Results Fundus photographs were taken at $8.01 \pm 2.22$ years of age for the preterm group and $8.01 \pm 2.13$ years of age for the control group. The mean gestational age of the preterm group was $27^{4} / 7$ weeks (range, 24-34 weeks). The preterm group had significantly larger $\mathrm{C} / \mathrm{D}$ ratio and smaller $\mathrm{A} / \mathrm{V}$ ratio (mean \pm standard deviation: $0.46 \pm 0.12$ and $0.59 \pm 0.08$, respectively) than the control group $(0.36 \pm 0.07$ and $0.68 \pm 0.07$, respectively) after spherical equivalent refractive error was adjusted.

Conclusions Preterm birth is significantly associated with larger $\mathrm{C} / \mathrm{D}$ ratio and smaller A/V ratio. These findings show the effect of preterm birth on the development of optic disc and retinal vessel development.

Eye (2015) 29, 1167-1172; doi:10.1038/eye.2015.99; published online 19 June 2015

\section{Introduction}

Preterm infants have a high risk of perinatal disease of the central nervous and vascular systems. ${ }^{1,2}$ Retinopathy of prematurity (ROP) is a disorder of vascular proliferation that occurs in the retinal development of preterm, low-birthweight infants. ${ }^{3}$ During follow-up of children previously screened for ROP, we received the impression that they often had a large cup-todisc (C/D) ratio and a small arteriole-to-venule (A/V) ratio. In 2010, Wikstrand et al ${ }^{4}$ reported the association of low birth weight and weight at gestational age (GA) of 32 weeks with large area of the optic disc cup in preterm birth. Plus disease, an indicator of ROP severity, may present a constellation of signs including retinal arterial tortuosity and venous dilation at the posterior pole, vitreous haze, and iris rigidity. ${ }^{5}$ In several studies, investigators have used vessel width to determine the presence of severe ROP or to predict later disease. ${ }^{6-8}$ For instance, Grunwald $e a^{8}{ }^{8}$ documented that the rate of increase in retinal venule width was useful in identifying infants at risk for severe ROP. Meanwhile, one morphometric study performed on fundus photographs from the selected group of preterm birth with a GA $<32$ weeks showed no optic disc changes but an abnormal vascular pattern compared with the control group of fullterm birth. ${ }^{9}$ Jacobson et $a l^{10}$ obtained fundus photographs from a group of preterm birth subjects with periventricular leucomalacia, which demonstrated large cups in normal-sized optic discs, suggestive of a variant of optic nerve hypoplasia. After that, Hellström et al ${ }^{11}$ demonstrated that the median optic disc rim area was significantly smaller in preterm birth subjects compared with a control group.

To our knowledge, no case-control study of age- and sex-matched controls has been performed on the $\mathrm{C} / \mathrm{D}$ ratio and $\mathrm{A} / \mathrm{V}$ ratio of preterm birth. Such children are of particular interest as they have demonstrated a high frequency of visual dysfunction. ${ }^{12}$ The purpose of this study was to investigate the influence of preterm birth on the optic disc and retinal vessels. The present study explored differences of $C / D$ ratio and $A / V$ ratio between preterm birth and age- and sex-matched controls.

\section{Materials and methods}

\section{Subjects}

This retrospective study was conducted according to the Declaration of Helsinki, after approval by the Institutional Review Board of the Samsung Medical Center in Korea. Among preterm births who visited the Pediatric
${ }^{1}$ Laboratory of Vascular Biology and Stem Cells, Graduate School of Medical Science and Engineering, Korea Advanced Institute of Science and Technology (KAIST), Daejeon, Republic of Korea

${ }^{2}$ Department of Ophthalmology, Samsung Medical Center, Sungkyunkwan University School of Medicine, Seoul, Republic of Korea

Correspondence: SY Oh, Department of Ophthalmology, Samsung Medical Center, Sungkyunkwan University School of Medicine, 50 Irwon-dong, Seoul 135710, Republic of Korea Tel: +82 23410 3566; Fax: +82 234100074. E-mail: syoh@skku.edu

Received: 30 August 2014 Accepted in revised form: 29 April 2015 Published online: 19 June 2015 
Ophthalmology and Strabismus Clinic, Department of Ophthalmology, Samsung Medical Center, between September 2011 and February 2013, we selected fundus color photographs from 83 eyes of 42 patients except images which had poor image quality insufficient to measure both C/D and A/V ratios exactly. For each patient, we looked for a full-term control individually matched in age and sex, and then 83 eyes of 42 children constituted a control group for evaluation of C/D and $\mathrm{A} / \mathrm{V}$ ratios. The control group consisted of subjects who visited the clinic for regular eye health check-up. All controls had no history of any disease likely to have had effect on eye development from birth until their eye examination. Especially, they had no history of any neurological disease or elevated intraocular pressure. All subjects in the preterm and control group included in this study have the same race and ethnicity as Korean.

\section{Imaging acquisition and analysis}

Fundus color photographs were taken with a model IX50 camera (Topcon, Paramus, NJ, USA) from 38 eyes in the preterm group and 36 eyes in the control group between September 2011 and September 2012. As Optos Panoramic 200 system (Optos plc, Dunfermline, UK) was introduced to our clinic since October 2012, fundus images taken with this system were obtained from 45 eyes in the preterm group and 47 eyes in the control group between October 2012 and February 2013. Images were taken through a dilated pupil from both eyes of 84 objects. Using the IX50 camera, $30^{\circ}$ retinal photographs were taken, and photographs up to $200^{\circ}$ of the retina were taken using the Optos Panoramic 200 system. Photographic methods were standardized with written protocols, and photographer performance was monitored with periodic feedback to assure optimal performance. At least one image was obtained from each eye of all 84 objects.

Fundus camera images were exported to ImageJ version 1.45 (Rasband, W.S., US National Institutes of Health, Bethesda, MD, USA) for measurements of photographs to be made in pixels. This expedites the estimation of ratios (for example, arteriole to venule ratio) and nullifies the need for camera magnification factors to be calculated. Images were obtained from both eyes of each participant except two eyes, which had fundus images that were inadequate for acquiring viable measurements.

\section{Cup-to-disc ratio}

An area of the photograph containing the optic disc was assessed. Photographs taken with the IX50 camera were enlarged to $150 \%$ and the photographs taken with the Panoramic 200 system were enlarged to $300 \%$. As described previously, ${ }^{13}$ vertical cup and disc diameters were measured using a straight line measurement tool incorporated into the ImageJ program (Figure 1). Two measurements were gained and averaged for each diameter. The area of the optic disc was defined as the area within Elschnig's ring. ${ }^{14}$ The border between optic cup and neuroretinal rim was defined as the level at which the slope of the rim steepens on the basis of contour, not pallor. ${ }^{14}$ If the cup or disc outline was not distinctly visible in the photograph, we obtained measurements using the 'sharpen' application (7 eyes, $4.2 \%$ ). The most clearly defined photograph was selected for analysis in cases where more than one photograph was available for each eye.

\section{Arteriole-to-venule ratio}

As described previously, ${ }^{13,15}$ an arteriole and venule within $0.5-1$ disc diameter from the edge of the disc margin was evaluated. A width for the defined arteriole and venule diameters was assessed using the straight line measurement tool (Figure 2). Between the concentric circles along the vessel as defined in Figure 2, five nonoverlapping measurements were made and averaged for a ratio calculated. The magnification of the retinal photographs taken by the fundus camera has been described. ${ }^{16}$ The revised Knudtson-Parr-Hubbard formulas were used to standardize individual vessel calibers of each eye. ${ }^{17}$ These formulas summarize the retinal vessel diameters restricted to the six largest retinal
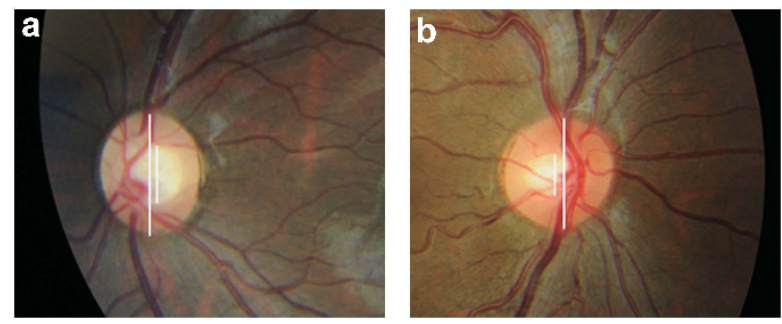

Figure 1 Example images of optic disc diameter and optic cup diameter measurements in the preterm group (a) and the control group (b).
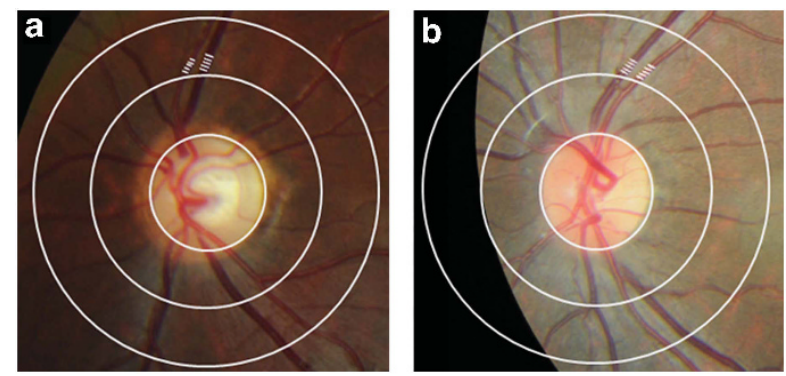

Figure 2 Example images of arteriole and venule diameter measurements in the preterm group (a) and the control group (b). 
vessels measured from the fundus photographs. ${ }^{17}$ The formulas used are $W_{\mathrm{c}}=0.88 \times\left(W_{\mathrm{a}}{ }^{2}+W_{\mathrm{b}}{ }^{2}\right)^{1 / 2}$ for arterioles and $W_{\mathrm{c}}=0.95 \times\left(W_{\mathrm{a}}{ }^{2}+W_{\mathrm{b}}{ }^{2}\right)^{1 / 2}$ for venules, in which $W_{\mathrm{c}}$ is the estimate of parent trunk arteriole or venule, $W_{\mathrm{a}}$ is the diameter of the narrower branch, and $W_{\mathrm{b}}$ is the diameter of the wider branch. ${ }^{17}$ After trunk variants of central retinal artery equivalent and central retinal vein equivalent were calculated using previously described iterative procedure, ${ }^{17}$ central retinal artery equivalent was divided by central retinal vein equivalent to obtain $\mathrm{A} / \mathrm{V}$ ratio. All measurements for both $\mathrm{C} / \mathrm{D}$ and $\mathrm{A} / \mathrm{V}$ ratios were made by a single observer (J.K.), and the intraobserver variability in the measurement of $C / D$ ratio was estimated.

\section{Statistical analyses}

Statistical analyses were applied to compare spherical equivalent refractive error and best spectacle corrected visual acuity (Log MAR) as well as C/D and A/V ratios between patient and control groups. To consider two eyes in one person, the method of generalized estimating equation was used. The correlation of spherical equivalent refractive error on $\mathrm{C} / \mathrm{D}$ and $\mathrm{A} / \mathrm{V}$ ratios was adjusted. Statistical analyses were performed with Geepack package R 3.0.1 (The R Foundation for Statistical Computing, Vienna, Austria). For all tests, a $P$-value $<0.05$ was considered statistically significant.

\section{Results}

\section{Clinical and demographic characteristics}

Eighty-three eyes of 42 patients in the preterm group and 83 eyes of 42 patients in the control group were included. In the preterm group, 67 eyes of 34 patients had previous history of ROP, on the other hand, 16 eyes of 8 patients had no ocular abnormalities. Among eyes with ROP, 50 eyes received laser photogoagulation, and cryotherapy was performed in 4 eyes. No history of intravitreal injection of anti-vascular endothelial growth factor was found. The mean GA of the preterm group was $27^{4 / 7}$ weeks (range, 24-34 weeks). In the preterm group, only one subject had a history of periventricular leucomalacia, and optic nerve hypoplasia was found in the fundus photograph of another one subject. In this study, optic nerve hypoplasia was limited to the definite cases, which showed small optic discs. No history of periventricular leucomalacia or optic nerve hypoplasia was shown in the subjects of the control group. Fundus photographs were obtained at an age of $8.01 \pm 2.22$ years (range, $3-14$ years) for the preterm group and $8.01 \pm 2.13$ years (range, 3-13 years) for the control group (Table 1). Forty-seven subjects $(56.6 \%)$ in both the preterm group and the control group were male (Table 1). The mean value of spherical equivalent refractive error in the preterm group was $-2.48 \pm 4.51$ (Table 1). In the control group, the mean value of spherical equivalent refractive error was $-0.80 \pm 2.35$ (Table 1). The value in the preterm group was significantly more myopic than that in the control group $(P=0.003$, Table 1$)$. Eyes in the control group had significantly better best-corrected visual acuity than those in the preterm group $(P<0.001$, Table 1$)$. Among 42 subjects in the preterm group, 36 patients had medical records of the neonatal intensive care units and pediatric clinics at the Samsung Medical Center, and remaining six neonates were outborn. Thirty patients $(83.3 \%)$ had suffered from respiratory distress syndrome and/or bronchopulmonary dysplasia, and the duration of oxygen therapy was $65.6 \pm 56.4$ days. Persistent patent ductus arteriosus was found in 24 subjects $(66.7 \%)$ and ductal ligation was performed in 10 cases. Only 3 patients (8.3\%) had a history of persistent pulmonary hypertension, and some subjects were diagnosed with left pulmonary arterial hypoplasia $(n=1)$, mitral regurgitation $(n=1)$ and atrial septal defect $(n=2)$. On the other hand, all subjects in the control group had no history of cardiac or pulmonary disorders that might have affected vascular diameter. Among some medications including calcium channel blockers, prostaglandin analogs, $\beta$-blockers, and oral corticosteroids, which might have influenced vascular diameter, ${ }^{18}$ beraprost sodium, a prostaglandin analog, was taken by one patient in the preterm group. In the control group, no one had a history of any drug associated with vessel width.

\section{Image parameters}

Table 2 shows means of $\mathrm{C} / \mathrm{D}$ and $\mathrm{A} / \mathrm{V}$ ratios in both groups. The mean $\mathrm{C} / \mathrm{D}$ ratio \pm standard deviation in the preterm group and the control group were $0.46 \pm 0.12$ and $0.36 \pm 0.07$, respectively. Before and after adjusted by spherical equivalent refractive error, the C/D ratio in the preterm group was significantly greater than that in the control group $(P<0.001)$. The intraclass correlation coefficient showing intraobserver variability represented

Table 1 Demographic and clinical characteristics of the preterm group and the control group

\begin{tabular}{lccr}
\hline & $\begin{array}{c}\text { Preterm } \\
\text { group }\end{array}$ & $\begin{array}{c}\text { Control } \\
\text { group }\end{array}$ & P-value \\
\hline No. of eyes & 83 & 83 & \\
Age, mean \pm SD, years & $8.01 \pm 2.22$ & $8.01 \pm 2.13$ & $1.000^{\mathrm{a}}$ \\
Sex: male to female & $47: 36$ & $47: 36$ & $1.000^{\mathrm{b}}$ \\
Spherical equivalent refractive & $-2.48 \pm 4.51$ & $-0.80 \pm 2.35$ & $0.003^{\mathrm{a}}$ \\
error, mean \pm SD, D & $0.24 \pm 0.29$ & $0.02 \pm 0.04$ & $<0.001^{\mathrm{a}}$ \\
BCVA, mean \pm SD, logMAR & &
\end{tabular}

Abbreviations: SD, standard deviation; D, diopter; BCVA, best corrected visual acuity. ${ }^{a}$ Univariate generalized estimating equation analysis. ${ }^{b} \chi^{2}$ test. 
Table $2 \mathrm{C} / \mathrm{D}$ ratio and $\mathrm{A} / \mathrm{V}$ ratio of the preterm group and the control group

\begin{tabular}{lccr}
\hline & Preterm group $(\mathrm{n}=83)$ & Control group $(\mathrm{n}=83)$ & P-value \\
\hline $\mathrm{C} / \mathrm{D}$ ratio, mean $\pm \mathrm{SD}$ & $0.46 \pm 0.12$ & $0.36 \pm 0.07$ & $<0.001^{\mathrm{a}}$ \\
$\mathrm{A} / \mathrm{V}$ ratio, mean $\pm \mathrm{SD}$ & $0.59 \pm 0.08$ & $0.68 \pm 0.07$ & $<0.001^{\mathrm{a}}$
\end{tabular}

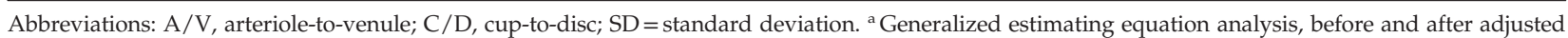
by spherical equivalent refractive error.

good reliability $(0.913 ; 0.820-0.953,95 \%$ confidence interval). The mean $\mathrm{A} / \mathrm{V}$ ratio \pm standard deviation in the preterm and the control group were $0.59 \pm 0.08$ and $0.68 \pm 0.07$, respectively. The $\mathrm{A} / \mathrm{V}$ ratio in the preterm group was significantly less than that in the control group regardless of the adjustment to the spherical equivalent refractive error $(P<0.001)$. In the subgroup analysis of the preterm group, both eyes with ROP $(N=67)$ and without previous ocular abnormalities $(N=16)$ had significant differences in $C / D$ and $A / V$ ratios compared with the control group $(P<0.001$ and $P<0.001$, respectively). In the correlation analysis, GA was marginally negatively correlated with C/D ratio (Pearson's $r=-0.402, P=0.071$ ) and positively correlated with A/V ratio (Pearson's $r=0.231, P=0.084)$.

\section{Discussion}

There have been a few previous studies about optic disc morphology in preterm birth compared with full-term birth. A previous morphometric study demonstrated no optic disc changes, except for the most preterm girls who had slightly lager disc areas. ${ }^{9}$ This finding is not in agreement with the present study. In this study, the mean $\mathrm{C} / \mathrm{D}$ ratio in the preterm group was significantly greater than that in the full-term group. This might be explained by the fact that the previous study was performed on a group of preterm birth with a higher mean GA of $29^{1 /} / 7$ weeks than those in the present study (mean GA of $27^{4} / 7$ weeks). ${ }^{9}$ Therefore, preterm birth in the present study could be less mature at birth and vulnerable to perinatal influences on the central nervous system. Previously, Hellström et $a l^{11}$ also reported that the median optic disc rim area was significantly smaller in the preterm birth with a mean GA of 27 weeks compared with a full-term control group. This finding is similar to our analysis. But, in their study, fundus photographs were obtained at 5.1-9.3 years of age for the preterm group and 2.6-19.6 years of age for the control group. We, on the other hand, observed fundus appearances at $8.01 \pm 2.22$ years of age (range, 3-14 years) for the preterm group and $8.01 \pm 2.13$ years of age (range, 3-13 years) for the control group. Park et al ${ }^{19}$ reported that mean C/D ratio increased by 0.0075 per year in term birth and rate of mean $C / D$ ratio progression was double in preterm birth. So, the fact that
Hellström et al ${ }^{11}$ did not use age-matched controls could confound the study results.

About retinal vascular abnormalities in preterm birth compared with full-term birth, Hellström et al ${ }^{9,11}$ reported that preterm birth had significantly increased tortuosity of retinal vessels and reduced number of vascular branching points compared with those of control subjects.

Meanwhile, Ghodasra et $a l^{3}$ enrolled eyes of 41 infants at risk for ROP, and demonstrated that eyes that eventually developed type 1 ROP showed a faster increase in width and tortuosity of retinal vessels compared with those that did not. In the study, type 1 ROP eyes had a greater rate of change in width for venule alone; the average rate of change in width for arterioles was not significantly different between groups. ${ }^{3} \mathrm{~A} / \mathrm{V}$ ratio is widely used in studies of the associations of retinal microvascular disease with systemic and ocular outcomes. ${ }^{20}$ In particular, retinal arterial tortuosity and venous dilation at the posterior pole are important findings to diagnose plus disease in ROP. ${ }^{5}$ To the best of our knowledge, the difference of $\mathrm{A} / \mathrm{V}$ ratio between preterm birth and full-tern birth matched with age and sex was not reported yet. In the present study, the mean A/V ratio in the preterm group was significantly less than that in the full-term group. As preterm birth has to adapt to a high extrauterine oxygen tension, the development of the vascular system may be considerably altered. ${ }^{3}$ An altered environment may lead to changes in stimulatory and inhibitory mechanisms that may affect morphology of arteriole and venule. ${ }^{3}$

In the present study, the mean value of spherical equivalent refractive error in the preterm group was significantly more myopic than that in the control group. Whether the correlation of spherical equivalent refractive error on $\mathrm{C} / \mathrm{D}$ ratio and $\mathrm{A} / \mathrm{V}$ ratio was adjusted or not, the mean $C / D$ ratio in the preterm group was significantly greater than that in the control group, and the mean $\mathrm{A} / \mathrm{V}$ ratio in the preterm group was significantly less than that in the control group. In 2004, Tong et al ${ }^{21}$ carried out a large study with 679 myopic subjects and 100 emmetropic subjects aged 8-13 years. They reported that C/D ratios were smaller in participants with myopia compared with those with emmetropia but $\mathrm{C} / \mathrm{D}$ ratios were not associated with severity of myopia. ${ }^{21}$ It is not apparent whether they adjusted potential confounding factors (that is, age, sex) in the analysis of the data. Further investigation about association between refractive error 
and $C / D$ ratio in children is needed to adjust confounding factors.

Compared with the control group, mean best-corrected visual acuity of eyes in the preterm group was significantly worse. ROP and neurological damage are often cited as major causes of the severe cases of decreased visual acuity in preterm birth. ${ }^{22}$ Dowdeswell et al demonstrated that the poor visual acuity of premature children was closely associated with their higher incidence of ocular pathology including abnormalities of the fundus and optic disc. ${ }^{23}$ In addition, it was reported that very preterm delivery with 31 weeks of mean GA generally had an effect on the potential for developing full visual acuity, also in individuals without evidence of early ROP. ${ }^{24}$ In the current study, among 83 eyes in the preterm group, 67 eyes had previous history of ROP and even 50 eyes received laser photocoagulation for treatment of ROP. Furthermore, although remaining 16 eyes had no history of ROP, they would also be influenced by preterm birth. Therefore, large $\mathrm{C} / \mathrm{D}$ ratio and small $\mathrm{A} / \mathrm{V}$ ratio would suggest the possibility of association between these parameters and hindered development of full visual acuity.

The present study had some limitations, including the retrospective nature of this study and the relatively small number of subjects. Having relatively small sample size might preclude achieving statistical significance at the 0.05 level, especially in the association between GA and imaging parameters. The fact that fundus photographs were taken by two different imaging modalities may distort the conclusion of the current study. However, among 83 matched pairs, 81 pairs consisted of subjects whose fundus images were taken with same imaging system. Only two remaining pairs were compared with image parameters originated from different imaging modalities. Therefore, the use of different imaging modalities would have little effect on the conclusion. Other optic disc parameters and vascular abnormalities were not analyzed in this study. But, we analyzed differences of $C / D$ ratio and $A / V$ ratio between preterm birth and age-, sex-matched controls. Until now, no casecontrol study of age- and sex-matched controls has been carried out on the $\mathrm{C} / \mathrm{D}$ ratio and $\mathrm{A} / \mathrm{V}$ ratio of preterm birth.

In conclusion, preterm birth with a mean age of $27^{4} / 7$ weeks (range, 24-34 weeks) had significantly greater mean $\mathrm{C} / \mathrm{D}$ ratio than the control group of full-term birth. The mean $\mathrm{A} / \mathrm{V}$ ratio in the preterm group was significantly less than that in the control group. These findings showed the effect of preterm birth on the development of optic disc and retinal vessels and possibly subsequent decreased visual acuity. In the future, a multicenter, randomized, controlled clinical trial for optic disc measurements and vascular morphology of preterm birth is necessary to fundamentally evaluate the influence of preterm birth on optic disc and retinal vessel development.

Summary

What was known before

- There have been a few previous studies about optic disc morphology and retinal vascular abnormalities in preterm birth compared with full-term birth.

What this study adds

- To our knowledge, no case-control study with age- and sex-matched controls has been performed on the C/D ratio and $\mathrm{A} / \mathrm{V}$ ratio of preterm birth.

- In the present study, the preterm group had significantly larger $\mathrm{C} / \mathrm{D}$ ratio and smaller $\mathrm{A} / \mathrm{V}$ ratio than the control group before and after spherical equivalent refractive error was adjusted.

\section{Conflict of interest}

The authors declare no conflict of interest.

\section{Authors contributions}

JK, KAP and SYO designed the study, collected and analyzed the data, and wrote the manuscript. DYC participated in data analysis and interpretation. All authors approved the final version of the manuscript.

\section{References}

1 The Committee for the Classification of Retinopathy of Prematurity. An international classification of retinopathy of prematurity. Arch Ophthalmol 1984; 102: 1130-1134.

2 De Reuck J, Chatta A, Richardson E. Pathogenesis and evolution of periventricular leukomalacia in infancy. Arch Neurol 1972; 27: 229-236.

3 Ghodasra DH, Thuangtong A, Karp KA, Ying GS, Mills MD, Wilson CA. The rate of change in retinal vessel width and tortuosity in eyes at risk for retinopathy of prematurity. J AAPOS 2012; 16: 431-436.

4 Wikstrand MH, Hård AL, Niklasson A, Hellström A. Birth weight deviation and early postnatal growth are related to optic nerve morphology at school age in children born preterm. Pediatr Res 2010; 67: 325-329.

5 Wilson CM, Cocker KD, Moseley MJ, Paterson C, Clay ST, Schulenburg WE et al. Computerized analysis of retinal vessel width and tortuosity in premature infants. Invest Ophthalmol Vis Sci 2008; 49: 3577-3585.

6 Rabinowitz MP, Grunwald JE, Karp KA, Quinn GE, Ying GS, Mills MD. Progression to severe retinopathy predicted by retinal vessel diameter between 31 and 34 weeks of postconception age. Arch Ophthalmol 2007; 125: 1495-1500.

7 Johnson KS, Mills MD, Karp KA, Grunwald JE. Quantitative analysis of retinal vessel diameter reduction after photocoagulation treatment for retinopathy of prematurity. Am J Ophthalmol 2007; 143: 1030-1032. 
8 Grunwald L, Mills MD, Johnson KS, Karp KA, Quinn GE, Ying GS et al. The rate of retinal vessel dilation in severe retinopathy of prematurity requiring treatment. Am J Ophthalmol 2009; 147: 1086-1091.

9 Hellström A, Hård AL, Chen Y, Niklasson A, AlbertssonWikland K. Ocular fundus morphology in preterm children. Influence of gestational age, birth size, perinatal morbidity, and postnatal growth. Invest Ophthalmol Vis Sci 1997; 38: 1184-1192.

10 Jacobson L, Hellström A, Flodmark O. Large cups in normal sized optic discs: a variant of optic nerve hypoplasia in children with periventricular leukomalacia. Arch Ophthalmol. 1997; 115: 1263-1269.

11 Hellström A, Hård AL, Svensson E, Niklasson A. Ocular fundus abnormalities in children born before 29 weeks of gestation: a population-based study. Eye (Lond) 2000; 14: 324-329.

12 Dowdeswell HI, Slater AM, Broomhall I, Tripp J. Visual deficits in children born at less than 32 weeks' gestation with and without major ocular pathology and cerebral damage. Br J Ophthalmol. 1995; 79: 447-452.

13 McClelland JF, O'Donoghue L, McIntyre M, Saunders KJ. Cup-to-disc and arteriole-to-venule ratios in children aged 6-7 and 12-13 years. Ophthalmic Physiol Opt. 2012; 32: 31-38.

14 Britton RJ, Drance SM, Schulzer M, Douglas GR, Mawson DK. The area of the neuroretinal rim of the optic nerve in normal eyes. Am J Ophthalmol 1987; 103: 497-504.

15 Leung H, Wang JJ, Rochtchina E, Tan AG, Wong TY, Klein R et al. Relationships between age, blood pressure, and retinal vessel diameters in an older population. Invest Ophthalmol Vis Sci 2003; 44: 2900-2904.
16 Bengtsson B, Krakau CE. Correction of optic disc measurements on fundus photographs. Graefes Arch Clin Exp Ophthalmol 1992; 230: 24-28.

17 Knudtson MD, Lee KE, Hubbard LD, Wong TY, Klein R, Klein BE. Revised formulas for summarizing retinal vessel diameters. Curr Eye Res 2003; 27: 143-149.

18 Howard KP, Klein BE, Dreyer JO, Danforth LG, Klein R. Cross-sectional associations of medication and supplement use with retinal vascular diameter in the Beaver Dam Eye Study. JAMA Ophthalmol 2014; 132: 23-31.

19 Park HJ, Hampp C, Demer JL. Longitudinal study of optic cup progression in children. J Pediatr Ophthalmol Strabismus 2011; 48: 151-156.

20 Liew G, Sharrett AR, Kronmal R, Klein R, Wong TY, Mitchell $\mathrm{P}$ et al. Measurement of retinal vascular caliber: issues and alternatives to using the arteriole to venule ratio. Invest Ophthalmol Vis Sci 2007; 48: 52-57.

21 Tong L, Saw SM, Chua WH, Luu C, Cheng B, Yeo I et al. Optic disk and retinal characteristics in myopic children. Am J Ophthalmol 2004; 138: 160-162.

22 O'Connor AR, Wilson CM, Fielder AR. Ophthalmological problems associated with preterm birth. Eye (Lond) 2007; 21: 1254-1260.

23 Dowdeswell HJ, Slater AM, Broomhall J, Tripp J. Visual deficits in children born at less than 32 weeks' gestation with and without major ocular pathology and cerebral damage. Br J Ophthalmol 1995; 79: 447-452.

24 Fledelius HC. Pre-term delivery and subsequent ocular development. A 7-10 year follow-up of children screened 1982-84 for ROP. 4) Oculometric - and other metric considerations. Acta Ophthalmol Scand 1996; 74: 288-293. 